\title{
Analytical modelling of rail track to account for nonlinear properties of structure
}

\author{
Piotr Koziol ${ }^{1, *}$ \\ ${ }^{1}$ Cracow University of Technology, Faculty of Civil Engineering, ul. Warszawska 24, 31-155 Kraków, Poland
}

\begin{abstract}
It is known that nonlinear and stochastic properties of structures should be taken into account in the analysis of their dynamic behaviour. This statement remains valid for railway infrastructure, where trains acting on rail track cause their strong dynamic variations. It becomes even more important when one deals with high frequencies and speeds of trains. The recent HSR networks need new technologies and materials in order to increase train speeds and, in the same time, to protect the environment against negative factors produced by operational lines. Therefore all important aspects of new railway constructions should be analysed, especially in terms of mechanical vibrations and noise attenuation. The elements of rail tracks, such as sleepers, under sleeper pads, rail dampers, fastening systems and others, have nonlinear properties. Their influence on dynamic behaviour of railway tracks is still insufficiently researched. For this purpose, various analytical models are proposed which can give a new insight into detailed dynamic characteristics of such structures.
\end{abstract}

\section{Introduction}

Several simplified analytical models of beam foundation structures can be found in the literature $[1,2]$. Those based on the dynamic equations of beams resting on viscoelastic foundation are the simplest ones used for the analysis of rail tracks [3, 4]. One should mention one-layer models representing rails resting on trackbed [4], two-layer models where the layers of rails and sleepers are described by joint differential equations [3] and multi-layered models with the beam resting on layered foundation (in particular on a half-space) [5]. In this last case, the layers of solid are described by dynamic equations of ground motion. This approach allowed to estimate and analyse critical velocities of loads travelling along the surface but also inside layer which is important for the analysis of underground transport [6, 7]. Inclusion of nonlinear properties of structures in these models leads to more complicated calculations needed to solve the systems. Classical approaches appear inadequate when one analyses dynamic mechanical systems with nonlinear factors and therefore new methods are sought. New subject of computational analysis arose from this need. This subject describes so called semi-analytical methods which are purely analytical tools combined with analytical approximations, such as series or sequences of functions, all of them without numerical contribution [8, 9]. One might say that it is a part of wider class of computational approaches, called hybrid methods. Here belong also numerical tools (based on discretisation of the investigated domain) enriched by analytical computations (e.g. Finite Element Method - FEM). The FEM is nowadays the most popular approach to the analysis of complex dynamic systems. Nevertheless, smart and simple analytical solutions make possible effective parametrical analysis without big computational effort using commercial computer programs. In order to achieve this aim (i.e. semi-analytical solution allowing efficient study), one must build mathematical model aimed at examining specific phenomena. The models mentioned in the introduction are devoted to the analysis of vertical vibrations of rail tracks $[2-4,7,8]$. Application of semianalytical methods based on Adomian's decomposition [10] and wavelet based analytical approximation [2] using wavelet filters [11] allowed their detailed analysis confirmed experimentally in some cases $[4,12]$. Further models modifications need however also improvements of applied solution methods, especially in terms of convergence and possible errors estimation.

\section{Models}

The most often analysed models related to rail track are usually based on more general mechanical multi-beam systems. The simplest model of rail track vertical vibrations can be described by the dynamic equation of the Euler-Bernoulli beam resting on viscoelastic foundation $[3,4]$ :

$$
\begin{gathered}
E I \frac{\partial^{4} w_{r}}{\partial x^{4}}+N \frac{\partial^{2} w_{r}}{\partial x^{2}}+m_{r} \frac{\partial^{2} w_{r}}{\partial t^{2}}+ \\
+c_{r} \frac{\partial w_{r}}{\partial t}+k_{L} w_{r}+k_{N} w_{r}^{3}=P(x, t)
\end{gathered}
$$

In this equation the following notation is used: $E I\left[\mathrm{~N} / \mathrm{m}^{2}\right]$ - a bending stiffness of rail steel; $N[\mathrm{~N}]$ - an axial force in rail;

* Corresponding author: pkoziol@pk.edu.pl 
$m_{r}[\mathrm{~kg} / \mathrm{m}]$ - a rail unit mass;

$k_{L}\left[\mathrm{~N} / \mathrm{m}^{2}\right]$ - a rail foundation stiffness (linear part);

$c_{r}\left[\mathrm{Ns} / \mathrm{m}^{2}\right]$ - a viscous damping of rail foundation;

$k_{N}\left[\mathrm{~N} / \mathrm{m}^{4}\right]$ - a nonlinear part of foundation (trackbed) stiffness.

The right hand side of equation (1) describes the load generated by train, acting on rail and moving along it with constant speed. This load can be modelled in various way, as a concentrated force, a load distributed on some interval with determined density or a set of forces constant or varying in time (usually harmonically) [3, 4, 6-8, 12]. One should note that the load considered in this paper represents only the weight of train and additional dynamic parts coming from irregularities of track (especially imperfections of the rolling rail head surface). The influence of contact forces is omitted.

Other more advanced models of rail track are built on the basis of so called multibeam systems. The theoretical double beam model assumes that two infinitely long beams are connected by viscoelastic layer and the whole system rests on viscoelastic foundation [13]. In terms of railway engineering, it can represent rail track, where the first beam shows rails behaviour and the second beam, in which the bending stiffness is neglected, describes a layer of sleepers as a rigid body $[3,8,12]$ :

$$
\begin{gathered}
E I \frac{\partial^{4} w_{r}}{\partial x^{4}}+m_{r} \frac{\partial^{2} w_{r}}{\partial t^{2}}+c_{r}\left(\frac{\partial w_{r}}{\partial t}-\frac{\partial w_{s}}{\partial t}\right)+ \\
+k_{r}\left(w_{r}-w_{s}\right)-k_{N} w_{s}{ }^{3}=P(x, t) \\
m_{s} \frac{\partial^{2} w_{s}}{\partial t^{2}}+c_{s} \frac{\partial w_{s}}{\partial t}+k_{s} w_{s}-c_{r}\left(\frac{\partial w_{r}}{\partial t}-\frac{\partial w_{s}}{\partial t}\right)- \\
-k_{r}\left(w_{r}-w_{s}\right)+k_{N s} w_{s}{ }^{3}=0
\end{gathered}
$$

Here, the index $r$ denotes rails and the index $s$ is used for sleepers layer.

The model given by equations (2a-b) includes also nonlinear part of foundation stiffness which leads to necessity of using Adomian procedure and wavelet approximation. This model gives reliable results concerned with the amplitude of vertical vibrations and also velocity and acceleration of these vibrations, along with character of time history in terms of function shapes.

Another step in construction of more realistic, but still simplified analytical models, related to rail track analysis is inclusion of nonlinearity in the layer between rails and sleepers. This property is also confirmed by experimental measurements and it is responsible for nonlinear features (mainly stiffness) of the fastening system. Equations (3ab) describe such a system. Its solution and analysis remains an open problem so far:

$$
\begin{gathered}
E I \frac{\partial^{4} w_{r}}{\partial x^{4}}+m_{r} \frac{\partial^{2} w_{r}}{\partial t^{2}}+c_{r}\left(\frac{\partial w_{r}}{\partial t}-\frac{\partial w_{s}}{\partial t}\right)+ \\
+k_{r}\left(w_{r}-w_{s}\right)+k_{N r} w_{r}{ }^{3}-k_{N s} w_{s}{ }^{3}=P(x, t) \\
m_{s} \frac{\partial^{2} w_{s}}{\partial t^{2}}+c_{s} \frac{\partial w_{s}}{\partial t}+k_{s} w_{s}-c_{r}\left(\frac{\partial w_{r}}{\partial t}-\frac{\partial w_{s}}{\partial t}\right)- \\
-k_{r}\left(w_{r}-w_{s}\right)+k_{N s} w_{s}{ }^{3}-k_{N r} w_{r}{ }^{3}=0
\end{gathered}
$$

This newly formulated model includes two nonlinearities; the first one $k_{N S}$ related to nonlinear part of foundation stiffness (trackbed) and the second one $k_{N r}$ which describes nonlinear stiffness of fastening system.

Appearance of two nonlinear factors leads to necessity of careful checking of all features of the model on the basis of extensive parametrical analysis. Before that, one must find effective solution method. Due to construction similar to previous systems, one can suppose that semianalytical techniques developed previously (Eqs. (1) and (2a-b)) might be sufficient for solving the model defined by equations (3a-b). However, several approximations appearing in this procedure are at least doubled in here and therefore existing convergence conditions might be inappropriate. The nonlinearities along with estimating procedures (Adomian series, wavelet approximation) and, additionally, with random representation of the load associated with train passage, might lead to instabilities, both the mathematical and structural. Detailed analysis of these issues is left for further work.

Before that, theoretical model should be analysed in order to find its applicability range. Therefore other models, such as a double-beam system with nonlinear layer between beams, resting on viscoelastic foundation (Eqs. (4a-b)), are formulated, solved and analysed for a wide system of parameters. These results will allow direct transition of the obtained solutions to railway engineering applications.

$$
\begin{gathered}
E I_{1} \frac{\partial^{4} w_{1}}{\partial x^{4}}+m_{1} \frac{\partial^{2} w_{1}}{\partial t^{2}}+c_{1}\left(\frac{\partial w_{1}}{\partial t}-\frac{\partial w_{2}}{\partial t}\right)+ \\
+k_{1}\left(w_{1}-w_{2}\right)+k_{N} w_{1}{ }^{3}=P(x, t) \\
E I_{2} \frac{\partial^{4} w_{2}}{\partial x^{4}}+m_{2} \frac{\partial^{2} w_{2}}{\partial t^{2}}+c_{2} \frac{\partial w_{2}}{\partial t}+k_{2} w_{2}- \\
c_{1}\left(\frac{\partial w_{1}}{\partial t}-\frac{\partial w_{2}}{\partial t}\right)-k_{1}\left(w_{1}-w_{2}\right)-k_{N} w_{1}{ }^{3}=0
\end{gathered}
$$

The model defined by equations (4a-b) is already successfully solved in case of two distributed forces harmonically varying in time with constant frequency, moving along the upper beam with some distance one from another [14]. The single force is defined by the following formula:

$$
P(x, t)=\frac{Q}{r} \cos ^{2}\left(\frac{\pi(x-V t)}{2 r}\right) H\left(r^{2}-(x-V t)^{2}\right)
$$

where $Q[\mathrm{~N}]$ is the value of force, $r[\mathrm{~m}]$ is the span of load, $H($.$) is the Heaviside function and V[\mathrm{~m} / \mathrm{s}]$ is the force velocity. In the next section, some discussion with regard to modelling of moving load is presented.

Various modifications of the described models can be made. The most interesting seems to be the replacement of the Euler-Bernoulli beam by the Timoshenko beam, especially in the case of double beam system. Some results obtained for the model of Timoshenko beam resting on nonlinear foundation and subjected to moving load or a set of forces, along with partial comparative study with regard to the Euler-Bernoulli beam investigation, were presented in published papers, e.g. $[15,16]$.

In this study, the following dynamic equation of Timoshenko beam is used:

$$
m_{b} \frac{\partial^{2} w}{\partial t^{2}}+C_{d} \frac{\partial w}{\partial t}-S \frac{\partial^{2} w}{\partial x^{2}}+S \frac{\partial \psi}{\partial x}+k_{L} w+
$$




$$
\begin{gathered}
+k_{N} w^{3}=P(x, t) \\
J \frac{\partial^{2} \psi}{\partial t^{2}}-E I \frac{\partial^{2} \psi}{\partial x^{2}}+S \psi-S \frac{\partial w}{\partial x}=0
\end{gathered}
$$

The symbols used in these formulas denote: $w[\mathrm{~m}]$ - a transverse displacement; $\psi$ - angular rotation of the cross-section;

$x[\mathrm{~m}]$ - a space coordinate in a direction along the beam; $t[\mathrm{~s}]$ - a time variable;

$k_{N}\left[\mathrm{~N} / \mathrm{m}^{4}\right]$ - a nonlinear part of foundation stiffness;

$k_{L}\left[\mathrm{~N} / \mathrm{m}^{2}\right]$ - a linear coefficient of foundation stiffness;

$E[\mathrm{~Pa}]$ - the Young's modulus;

$G[\mathrm{~Pa}]$ - the shear modulus;

$\rho\left[\mathrm{kg} / \mathrm{m}^{3}\right]$ - the mass density of beam;

$C_{d}\left[\mathrm{Ns} / \mathrm{m}^{2}\right]$ - the viscous damping of foundation,

$A\left[\mathrm{~m}^{2}\right]$ - the cross-section area;

$I\left[\mathrm{~m}^{4}\right]$ - the moment of inertia of plane area;

$\kappa$ - the shear correction factor;

$m_{b}=\rho A[\mathrm{~kg} / \mathrm{m}]$ - the mass of beam per unit length;

$S=\kappa A G[\mathrm{~N}]$ - the shear stiffness;

$E I\left[\mathrm{Nm}^{2}\right]$ - the beam flexural rigidity;

$J=\rho I[\mathrm{~kg} \mathrm{~m}]-$ the mass moment of inertia.

Because the beam is infinitely long, the boundary conditions should guarantee that the displacement, the bending moment, the shear force and the slope of the beam curvature tend to zero. This physically means that the energy produced by the moving load dissipate in some distance from the source of disturbance.

One should underline that various Timoshenko equations can be found in the literature, depending on investigated problems and features of the system [16].

\section{The load and its randomness}

The load generated by train can be modelled as a sum of three important parts:

$$
P(x, t)=P_{C}(x, t)+P_{V}(x, t)+P_{S}(x, t)
$$

where

$$
P_{C}(x, t)=\sum_{l=0}^{L} \frac{\mathrm{Q}}{r} d(x, t) H\left(r^{2}-\left(x-V t-s_{l}\right)^{2}\right)
$$

is a part of load constant in time, associated with the weight of train,

$$
\begin{gathered}
P_{V}(x, t)= \\
\sum_{l=0}^{L} \frac{\Delta Q}{r} e^{i \omega t} d(x, t) H\left(r^{2}-\left(x-V t-s_{l}\right)^{2}\right)
\end{gathered}
$$

is a dynamic part generated by regular imperfections of rail surface (it can be also deflection of rails between sleepers in the case of classical railway track) and $P_{S}(x, t)$ denotes stochastic part of load, generated by random conditions (when imperfections produce undetermined vibrations).

The following notation is used in Eq. (7)-(9): $H($.$) is$ the Heaviside function, $2 r$ is the span of single load, $s_{l}$ is the distance of axles from the front of the load (or the distance between forces associated with wheels) $L$ is the number of axles, $\omega$ is the frequency of load, $V$ is the speed of train and $Q$ is the force associated with single axle. The load density $d(x, t)$ can be modelled in various ways. It can have e.g. rectangular shape, Gauss or cosine functions, or the following representation:

$$
d(x, t)=\cos ^{2}\left(\frac{x-V t-s_{l}}{2 r}\right)
$$

which allows to alleviate doubts related to regularity of solution of differential equations (this is smooth function, so it has derivatives of all orders).

In previous papers, a special draw procedure of rail head surface irregularities was described [17, 18]. This procedure, although limited to specific imperfections, was associated with technical requirements of railways. It allowed to calculate acceleration of random vibrations generated by stochastic geometrical surface conditions and, consequently, to estimate the changes of random force appearing during train passage.

Figure 1 shows examples of such acceleration obtained by using the mentioned draw procedure. One should underline that this method gives also direct analytical formula describing the random part of load that can be introduced in Mathematica codes used for solving the system.
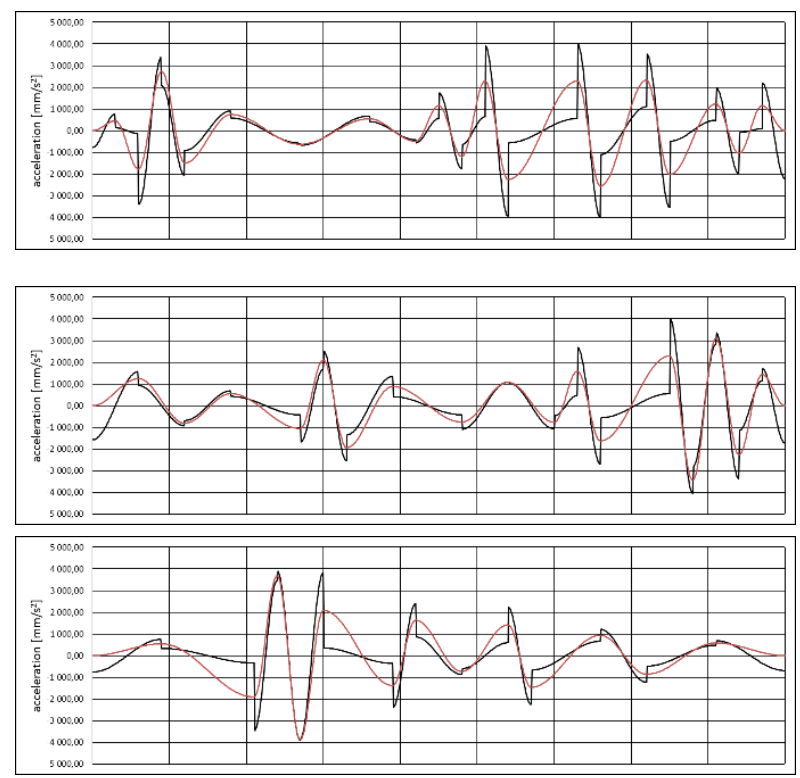

Fig. 1. Acceleration of random vibrations: second derivative of piecewise function describing random irregularities - black line, cosine approximation - red line (the length of beam: $9 \mathrm{~m}$; the length of intervals with different draw conditions: $6 \mathrm{~m}$ and $3 \mathrm{~m}$ ).

Compared to previous studies, in this paper, two regions of the beam are considered. In each of these regions, different conditions for irregularities draw procedure are assumed. This means different physical properties of imperfections in each interval. This is done due to importance of investigation of structure behaviour at the moment of crossing the border between these intervals by the moving load. Sudden change of rail track stiffness or its general quality are very important aspects of railway dynamics analysis. The developed model might help in the investigations of phenomena appearing in such situations, especially when one deals with HSR.

The randomly selected irregularities of the rail surface are approximated by cosine function which gives the piecewise function (Fig. 2). Then, after double 
differentiation with respect to the time variable, it must be again approximated by continuous function. This is due to a lack of derivatives at the ends of intervals, where the function changes its formula. The red line shows this approximation of second derivative made with cosine function (Fig. 3). It represents variation of vibration acceleration associated with randomly chosen irregularities of rail head rolling surface. This variation is described by a piecewise function which can be directly used in Mathematica codes.

The conditions of random selection of beam surface (rail head rolling surface) irregularities in further calculations are as follows:

1. The length of beam fragment with ,surface imperfections" is finite $(6 \mathrm{~m})$.

2. The step of draw in $x$ direction is fixed, although the code gives possibility to change it independently in separated sections.

3. The amplitude of imperfection is limited (finite).

4. The amplitude increase between two consecutively selected points is limited (and fixed).

5. The amplitude of imperfection is randomly chosen under condition that all above limitations are kept and all irregularities are below horizontal line (zero level).

6. The length of the beam with ,surface imperfections": $6 \mathrm{~m}$ (divided for two regions: 3 $\mathrm{m}$ and $3 \mathrm{~m}$ );

7. The step of draw in $x$ direction: $0.2 \mathrm{~m}$;

8. The amplitude of imperfection: $0.05 \mathrm{~mm}$ in the first region and $0.5 \mathrm{~mm}$ in the second region;

9. The amplitude increase: $0.02 \mathrm{~mm}$ in the first region and $0.2 \mathrm{~mm}$ in the second region.

Figure 2 shows examples of randomly generated irregularities along with their cosine and Fourier approximations. In Fig. 3, one can see the vibration accelerations associated with each case presented in Fig. 2 , with the load speed equal to $120 \mathrm{~km} / \mathrm{h}$.

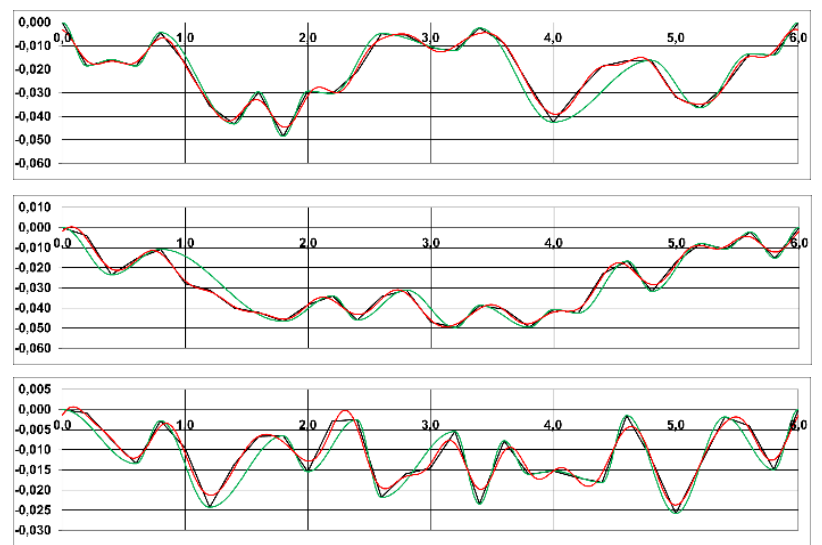

Fig. 2. Randomly selected irregularities of rail surface - black line, cosine approximation - green line, Fourier approximation red line (the length of beam: $6 \mathrm{~m}$; the length of each interval: 3 $\mathrm{m}$ and $3 \mathrm{~m}$ ).

This procedure will be used in further models extension, starting from the simplest model defined by equation (1). This relatively easy to solve system will allow recognition of all features important for correctness and reliability of the developed approach in terms of its railway applications. Therefore the set of parameters used in extended analysis will go beyond the frame of railway engineering, taking into account also cases from aerospace engineering, mechatronics or even more theoretical subjects. After detailed study and development of effective procedure of statistical analysis, the path for the method application to more complex systems will be open.
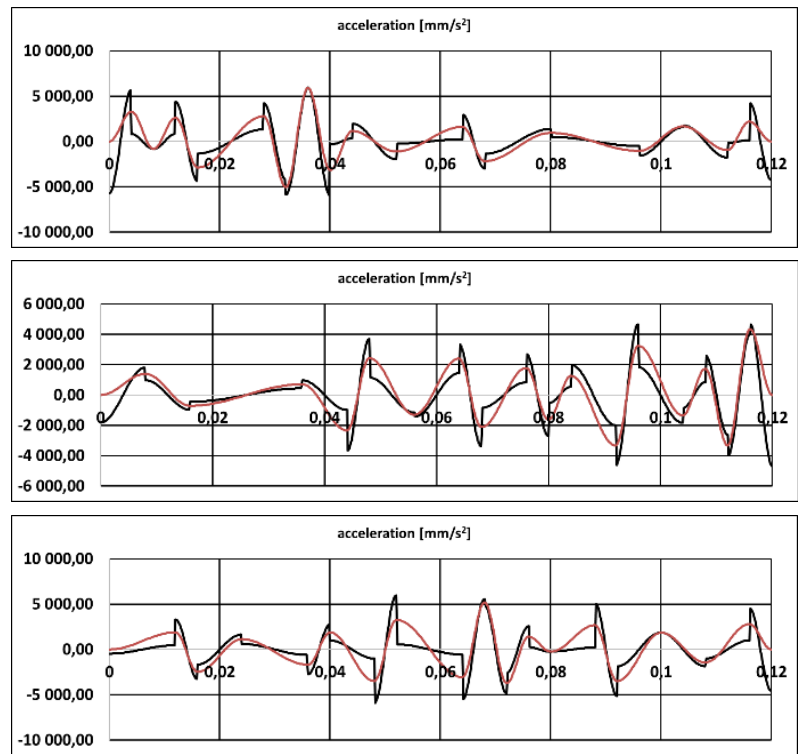

Fig. 3. Random acceleration of vibrations related to generated irregularities of rail surface (Fig. 2; comp. Fig. 1) - black line, cosine approximation - red line (the length of beam: $6 \mathrm{~m}$; the length of intervals: $3 \mathrm{~m}$ and $3 \mathrm{~m}$ ).

Piecewise function which can be used directly in Mathematica code has the following form:

1) $\mathrm{w}=$ Piecewise $\left[\left\{\left\{2.16246917843819 * 10^{\wedge}\right.\right.\right.$ $02 * \operatorname{Cos}[157.07963267949 * \mathrm{t}-0]$

$\left.2.16246917843819 * 10^{\wedge}-02,0<=\mathrm{t}<0.02\right\}$,

$\left\{1.55420497059822 * 10^{\wedge}\right.$

$02 * \operatorname{Cos}[392.699081698724 * \mathrm{t}$ -

$4.71238898038469]-2.77073338627815^{*} 10^{\wedge}$ _

$02,0.02<=\mathrm{t}<0.028\},\left\{1.86490118503571 * 10^{\wedge}{ }_{-}\right.$

$03 * \operatorname{Cos}[785.398163397449 * \mathrm{t}$ -

21.9911485751286]-0.014030185341835, $0.028<=\mathrm{t}<0.032\},\left\{7.94754326343536 * 10^{\wedge} \_\right.$

$03 * \operatorname{Cos}[392.699081698724 * \mathrm{t}-$

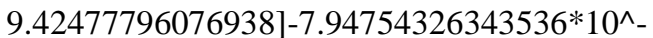

$03,0.032<=\mathrm{t}<0.04\}\}, 0]$

in the case of irregularities and

2) $\mathrm{w}=$ Piecewise $[\{\{732.586823020646 * \operatorname{Cos}[157.0$ $7963267949 * \mathrm{t}+3.14159265358979]+732.58682$ $3020646,0<=\mathrm{t}<0.02\}$,

$\{1619.37287752772 * \operatorname{Cos}[392.699081698724 * \mathrm{t}$ $-7.85398163397448]-154.199231486433$,

$0.02<=\mathrm{t}<0.028\}$,

$\{1480.78002225845 * \operatorname{Cos}[785.398163397449 * \mathrm{t}$

$-18.8495559215388]-292.79208675571$,

$0.028<=\mathrm{t}<0.032\}$, 


$$
\begin{aligned}
& \{593.993967751369 * \operatorname{Cos}[392.699081698724 * \mathrm{t} \\
& -12.5663706143592]+593.993967751369, \\
& 0.032<=\mathrm{t}<0.04\}\}, 0]
\end{aligned}
$$

in the case of acceleration.

For simplicity of the above formulas, the length of the random part of beam is fixed at 2 meters and the beam is divided in two even sections with random properties determined by described conditions 1-9.

Practical application of the developed procedure is planned for future work. There are two possible ways of investigation [17]:

- The first one is based on direct application of the formulas describing the random irregularities (acceleration) to previously developed analytical models. After solving, for a set of realisations, statistical analysis of the obtained solutions can be performed. In this case, each solution remains deterministic, although the load is generated randomly. Estimation of the force value related to the random part of load generated by the beam irregularities remains an open problem. One can do it through computational experiments and comparative studies involving measurements of real structures behaviour.

- The second one is based on statistical analysis of generated realisations of randomly chosen irregularities and building of stochastic function which describes indeterministic load. Inclusion of such a function in each of discussed models leads to formulation of problem in terms of stochastic differential equations. In this case, however, the problem becomes nonlinear and random which needs advanced techniques of stochastic analysis to be applied.

The both mentioned above approaches remain the main directions of further works.

\section{Convergence and stability}

Application of several approximations leads to problems associated with convergence of solutions and, consequently, their accuracy. This is important not only for obtaining proper solutions but also for the models applicability and, physically, for the analysis of structures stability.

There are several different approximation procedures used in semi-analytical solutions of models described in section 2. Each of them needs formulation of proper conditions for solutions accurate enough. Additional difficulty comes from the fact that all these approximations are mixed and used several times within computational algorithms. Therefore accuracy of computations must be controlled at each stage of solution.

For the Adomian decomposition needed for nonlinear term representation, the following convergence condition can be applied $[10,13]$ :

$$
0 \leq \propto_{j}<1 \text { for } j=0,1,2, \ldots
$$

where

$$
\propto_{j}= \begin{cases}\frac{\left\|w_{j+1}\right\|}{\left\|w_{j}\right\|} & \text { for }\left\|w_{j}\right\| \neq 0 \\ 0 & \text { for }\left\|w_{j}\right\|=0\end{cases}
$$

with the norm

$$
\left\|w_{j}\right\|=\max _{x}\left|\operatorname{Re}\left[w_{j}(x)\right]\right|
$$

for each term of Adomian series

$$
w(x)=\sum_{j=0}^{\infty} w_{j}(x) .
$$

It is shown $[2,7,15]$ that effective analysis can be carried out by using the $n$-th order of approximation:

$$
S_{n}^{w}(x)=\sum_{j=0}^{n} w_{j}(x) .
$$

The convergence condition (11) should be used for each function decomposed by Adomian series (e.g. in the case of two-layer model with nonlinearities in both layers, for upper and lower beams separately).

In the case of wavelet approximation, the criterion of the solution stability is checked and confirmed for several dynamic systems, including nonlinear beam-foundation structures subjected to moving loads. This issue is widely discussed in published papers, e.g. [13].

Stochastic systems are also very sensitive for variations of parameters and detailed analysis of the solution behaviour should be carried out in order to keep the stationary character of random processes.

The value of parameter describing nonlinear part of stiffness is a very important factor in the analysis of nonlinear systems defined in section 2 [19]. Its appropriate estimation usually decides about model applicability in terms of stability of structure.

\section{Conclusions}

Several models of rail track are described and discussed with special emphasis on computational techniques used for their solution. Previously defined two layer nonlinear model is extended by inclusion of additional nonlinear factor responsible for stiffness of fastening system. Special procedure for modelling of load generated by rail head surface irregularities is introduced with possibility of representation of several separated regions of beam (rail) with different random geometrical properties. A number of computational examples are presented for presentation of the developed method possibilities. Possible issues appearing in solving procedures are discussed, especially these associated with convergence of solutions obtained by using semi-analytical methods based on analytical approximations.

\section{References}

1. L. Fryba, Thomas Telford Ltd., London, (1999)

2. P. Koziol, VDM Verlag Dr. Müller, Saarbrucken, (2010)

3. W. Czyczula, P. Koziol, D. Kudla, S. Lisowski, J. Vib. Control 23, 18, 2989-3006 (2017)

4. P. Koziol, Mech. Syst. Signal Process., 79, 174-181 (2016) 
5. P. Koziol, M.M. Neves, SAV, 19, 1009-1018 (2012)

6. P. Koziol, C. Mares, SAV, 17 (4-5), 461-472 (2010)

7. P. Koziol, C. Mares, I. Esat, Int J Solids Struct. 45, 2140-2159 (2008)

8. P. Koziol, Proceedings of ICoEV2015, International Conference on Engineering Vibration, Ljubljana, Slovenia, 7-10 September 2015, paper 273, (2015)

9. Z. Dimitrovova, Proceedings of the $14^{\text {th }}$ International Conference on Vibration Engineering and Technology of Machinery (VETOMAC XIV), MATEC Web of Conferences 211, 11005 (2018)

10. G. Adomian, Kluwer Academic Publishers, Dordrecht, (1989)

11. S. Mallat, Academic Press Inc. Ltd, London (1998)

12. P. Koziol, Proceedings of the Fifteenth International Conference on Civil, Structural and Environmental Engineering Computing, Civil-Comp Press, Stirlingshire, UK, Paper 199 (2015)

13. P. Koziol, J. Theor. Appl. Mech., 52, 3, 687-697 (2014)

14. P. Koziol, R. Pilecki, Proceedings of the $14^{\text {th }}$ International Conference on Vibration Engineering and Technology of Machinery (VETOMAC XIV), MATEC Web of Conferences 211, 11008 (2018)

15. P. Koziol, Z. Hryniewicz, SAV, 19, 995-1007 (2012)

16. W. Czyczula, P. Koziol, D. Blaszkiewicz, SAV, Volume 2017, Article ID 2701715 (2017)

17. P. Koziol, D. Kudla, Proceedings of the MPSVA2018 conference, IOP Conference Series (in print)

18. P. Koziol, D. Kudla, C. Mares, Proceedings of the $14^{\text {th }}$ International Conference on Vibration Engineering and Technology of Machinery (VETOMAC XIV), MATEC Web of Conferences 211, 11007 (2018)

19. T. Dahlberg, Proceedings of Fifth International Conference on Structural Dynamics, Munich (2002) 